\title{
Evaluation of a disease specific rheumatoid arthritis self-management education program, a single group repeated measures study
}

Vironique Vermaak', N. Kathy Briffa', Bob Langlands'², Charles Inderjeeth ${ }^{2,4}$ and Jean McQuade ${ }^{3,5^{*}}$

\begin{abstract}
Background: Rheumatoid Arthritis is a progressive and disabling disease, predicted to increase in prevalence over the next 50 years. Self-management is acknowledged as an integral part in the management of chronic disease. The rheumatoid arthritis specific self-management program delivered by health professionals was developed by Arthritis Western Australia in 2006. The purpose of this study was to determine whether this program would achieve early benefits in health related outcomes, and whether these improvements would be maintained for 12 months.

Methods: Individuals with rheumatoid arthritis were referred from rheumatologists. Participants with co-existing inflammatory musculoskeletal conditions were excluded. All participants completed a 6-week program. Assessments occurred at baseline (8 weeks prior to intervention), pre-intervention, post-intervention, and 6 and 12 month follow ups. Outcomes measured included pain and fatigue (numerical rating scale, 0-10), depression and anxiety (hospital anxiety and depression questionnaire), health distress, and quality of life (SF-36 version 2).
\end{abstract}

Results: There were significant improvements in mean [SD] fatigue (5.7 [2.4] to 5.1 [2.6]), depression (6.3 [4.3] to 5.6 [3.9]) and SF-36 mental health (44.5 [11.1] to 46.5 [9.5]) immediately following intervention, with long term benefits for depression (6.3 [4.3] to 4.9 [3.9]), and SF-36 subscales mental health (44.5 [11.1] to 47.8 [10.9]), role emotional (41.5 [13.2] to 46.5 [11.8]), role physical (35.0 [11.0] to 40.2 [12.1]) and physical function (34.8 [11.5] to $38.6[10.7])$.

Conclusion: Participants in the program recorded significant improvements in depression and mental health post-intervention, which were maintained to 12 months follow up.

\section{Background}

Arthritis is one of the most prevalent chronic diseases in Australia [1], with nearly 0.5 million affected by the most severe type, rheumatoid arthritis (RA) [2]. Rheumatoid arthritis is an autoimmune disease of unknown aetiology [3, 4]. More women are affected by RA than men with $2.6 \%$ and $1.6 \%$ of the Australian population respectively affected [5]. By 2050, it is projected to affect 0.9 million Australians [1].

Rheumatoid arthritis causes progressive deterioration, with subsequent functional decline [6], leading to disability,

\footnotetext{
* Correspondence: jeanm@arthritiswa.org.au

${ }^{3}$ Arthritis and Osteoporosis Western Australia, Perth, Western Australia, Australia

${ }^{5}$ Health, Education \& Research Program Manager, Arthritis and Osteoporosis Western Australia, PO Box 34, Wembley WA 6913, 17 Lemnos St, Shenton Park, Western Australia 6008, Australia

Full list of author information is available at the end of the article
}

participation restrictions and impaired quality of life [7]. Individuals with RA experience greater psychological distress than the general population [8]. Furthermore, over $80 \%$ of individuals with RA have clinically important fatigue [9]. Pain, a cardinal symptom of RA, affects up to $84 \%$ of individuals [10], and negatively impacts on multiple aspects of life [11].

Self-management (SM) is increasingly being accepted as an integral part in the management of chronic disease [12]. Self-management interventions (SMIs) are patient-centred, problem focused and action-oriented [13, 14], addressing physical and psychosocial issues [15]. They utilise educational, behavioural and cognitive strategies $[12,14]$ to enhance patient participation in treatment [16].

Education programs (EPs) teaching SM skills are believed to be more effective than information-only EPs in 
improving clinical outcomes in RA patients $[17,18]$. However, reported benefits are equivocal and the benefits are temporary [17]. Systematic reviews have reported a trend towards small benefits from arthritis SM programs [16, 19-21]. Overall, SMIs improve knowledge, SM skills, self-efficacy (SE), and some aspects of health status [22, 23]. There is evidence supporting positive outcomes for disease-specific SMIs [16, 19, 20], including osteoarthritis [24]. Numerous studies have evaluated EPs and generic SMIs, however, only a small number have examined disease-specific SMIs. Disease-specific SMI are reported to produce better outcomes compared to generalised non-specific chronic disease SMIs [25, 26], and it is thought that this approach may be more beneficial for people with RA. The available evidence suggests that provision of tailor-made programs may be more beneficial for chronic disease management $[27,28]$ and thus identification of individual educational needs becomes imperative [29].

The RA SMI was developed by Arthritis Western Australia in 2006, using a participatory action research model, utilising the Plan, Do, Study, Act approach. The program was based on the findings of an educational needs assessment tool survey completed by a total of 157 RA patients from United Kingdom $(\mathrm{n}=125)$ and Western Australia $(n=32)$ in 2005 [30]. Findings demonstrated that people with RA have specific needs [30]. It was on this basis, that the RA SMI was developed. Participant needs catered for incorporated offering the program in a community setting with flexible delivery times including evenings and weekends as well as the content of the program. The intervention program is person-centred and based on SM principles, including cognitive behavioural therapy and SE constructs.

\section{Purpose of study}

The aim of this study was to determine whether the RA SMI would achieve immediate improvements in health related outcomes following program involvement, and whether these improvements would be maintained for 12 months after course completion.

\section{Methods}

\section{Study design}

A one group (within subjects) repeated measures study was used to determine change over time.

This study was approved by the Human Research Ethics Committee at Curtin University, Western Australia (PT0021, 2005). All participants provided written informed consent.

\section{Participants}

Patients with RA were recruited by referral from specialist rheumatologists in Western Australian public hospitals and private sectors. Inclusion criteria were clinical diagnosis of RA made by their treating rheumatologist, aged $\geq 18$ years, and the ability to speak and understand English. Participants with co-existing inflammatory musculoskeletal conditions were excluded. Diagnostic criteria were not specified but at the discretion of the clinical rheumatologist treating the patient.

\section{Outcome measures}

Outcome measures evaluated pain, fatigue, depression, anxiety, SE, health distress and health related quality of life. Participants completed a set of baseline (BL) questionnaires 8 weeks prior to the commencement of the program , then again one week prior to the first SM session (pre-intervention), one week after the 6 week program (post-intervention), with follow up periods at 6 and 12 months.

Pain and Fatigue were each measured via a ten-point incremental numerical rating scale. Numerical rating scales have adequate discriminative power for describing pain intensity [31], have good sensitivity [32] and the reliability is excellent $(\mathrm{r}=0.94)$ [33].

Depression and Anxiety were measured using the 14-item Hospital Anxiety and Depression scale, consisting of 7 anxiety and 7 depression items, scored separately [34] which is reliable (Cronbach's alpha 0.68 to 0.93 ) and valid [34, 35].

Self-Efficacy was measured using an 8-item questionnaire developed by Stanford University from the original 20-item arthritis SE scale [36]. The scale reflects how participants rate their certainty in performing tasks along a continuum. This scale is reliable and valid [36, 37] (http:// patienteducation.stanford.edu/research/searthritis.html).

Health Distress was measured using a 4 item questionnaire to assess the level of fear, worry and/or frustration each individual felt in relation to their disease [37]. Scores range from 0 to 5 with higher scores indicating more distress about health (http://patienteducation.stanford.edu/ research/searthritis.html).

Quality of life was measured using the Short-Form 36 Version 2 questionnaire (SF-36). The SF-36 consists of 36 questions and includes 8 component subscales reflecting physical and mental status [38], including physical function, role physical, bodily pain, general health, vitality, social function, role emotional and mental health. The SF-36 is a well-established outcome assessment in RA [39, 40] and is reliable and valid [41-43].

\section{Intervention}

The newly developed RA SMI program was offered to patients managed in a Rheumatology Outpatient Department of a tertiary hospital and in a community setting. Groups of 8-15 participants attended 1 session each week for 6 consecutive weeks, with each $2.5 \mathrm{~h}$ in duration. Attendance 
and assessment time-points were recorded. Modules are inter-related and cumulative building on previously learned information. The program was delivered by the same two health professionals. Uniformity of the RA program across and within groups was maintained by using scripted facilitator's manual.

The program content concentrated on disease-specific education including:

- Psychological impact and management strategies

- Fatigue management and energy conservation strategies

- Pain management

- Medications

- Exercise

- Posture, balance and falls prevention

- Nutrition

- Complimentary/alternative therapies

- Joint protection

- Osteoporosis

Personal development focused on individual weekly goal setting, problem solving, relaxation techniques, and cognitive behavioural therapy to assist in long term behavioural changes. Participants were encouraged to incorporate techniques learnt at each session into their activities of daily living, returning to discuss their experience next week.

\section{Data analysis}

Data was analysed using SPSS version 19. One way (repeated measures) analysis of variance (ANOVA) with time (baseline, pre-intervention, post-intervention, 6 and 12 months follow up) as the independent variable was utilised. Mauchly's Test of Sphericity was performed on all data. When the Mauchly's statistic was significant, with the sphericity assumption violated for the univariate approach to repeated-measures ANOVA, this was corrected with the Greenhouse-Geisser adjustment. Where tests of within-subjects comparisons were significant, implying a significant change over time, post-hoc linear contrasts were performed to compare each time point to the pre-intervention time point. Significant improvements between $\mathrm{BL}$ and pre-intervention were interpreted to indicate spontaneous improvement, suggesting that changes following the intervention may also be spontaneous. Statistical significance was inferred at $\mathrm{p}<0.05$.

\section{Results}

A total of 113 participants (96 [85 \%] female) with a mean (SD) age of 54 (13) were enrolled in the study. Ninety-six participants completed data collection at all time-points. Of these participants 92 (96\%) attended $80 \%$ or more of the intervention sessions.

All patients in the study were referred by and being treated by a clinical Rheumatologist at either a public hospital or private clinic. Treatment prescribed by these specialist physicians was at their discretion in consultation with the patient taking into account efficacy, tolerance, toxicity, cost, compliance and patient preference. At baseline, $43 \%, 76 \%$ and $13 \%$ of participants were taking steroids, disease modifying anti-rheumatic drugs (DMARDs) and biologics (bDMARDs), respectively. Steroid use decreased to $35 \%$ post-intervention and $32 \%$ at 12 months. DMARDs use increased to $79 \%$ at pre-intervention but stabilised at $76 \%$ post-intervention and at 12 months. The use of bDMARDs continually increased from $15 \%$ pre-intervention to $16 \%$ postintervention and $24 \%$ at 12 months. At 12 months, $59 \%$ of participants were taking symptomatic treatments such as paracetamol and NSAIDS.

\section{Fatigue and pain}

Fatigue and pain remained stable during the preintervention control period. The fatigue numerical rating score decreased significantly from a mean (SD) of 5.7 (2.4) pre-intervention to 5.1 (2.6) post-intervention, however, there was a small rebound to 5.2 (2.7) at 6 and 12 months which was no longer statistically significant (Table 1). Pain numerical rating score showed a small nonsignificant decrease throughout the course of the study.

\section{Anxiety and depression}

Levels of anxiety and depression remained stable during the control period. Anxiety had a non-significant reduction from a mean (SD) of 7.4 (3.9) to 6.9 (3.9) during the 6 week intervention. At 6 months, anxiety had decreased to 6.7 (4.0) and this level was maintained at 6.7 (4.5) at 12 months. Depression improved significantly postintervention and was maintained to 12 months (Table 1).

\section{Health distress}

Health distress lessened significantly during the preintervention control period. Post-intervention, only a small non-significant reduction was observed from mean (SD) 2.1 (1.1) to 2.0 (1.0). Further significant improvements in health distress were observed at 6 and 12 month (Table 1).

\section{Self-efficacy}

During the pre-intervention period, a significant improvement in SE was observed, with no incremental significant changes post-intervention. 
Table 1 Results for outcomes: fatigue, pain, anxiety, depression, health distress, self-efficacy, and SF-36 at baseline, pre-intervention, post-intervention, 6- and 12-months follow up. Data are mean (SD)

\begin{tabular}{|c|c|c|c|c|c|}
\hline Outcomes & Baseline & Pre-Ix & Post-Ix & 6 Months & 12 Months \\
\hline Fatigue (0-10) & $6.1(2.4)$ & $5.7(2.4)$ & $5.1(2.6)^{a}$ & $5.2(2.7)$ & $5.2(2.9)$ \\
\hline Pain (0-10) & $5.1(2.4)$ & $4.9(2.2)$ & $4.8(2.7)$ & $4.9(2.7)$ & $4.4(2.9)$ \\
\hline Anxiety (0-21) & $7.8(4.1)$ & $7.4(3.9)$ & $6.9(3.9)$ & $6.7(4.0)^{\mathrm{a}}$ & $6.7(4.5)$ \\
\hline Depression (0-21) & $6.1(3.8)$ & $6.3(4.3)$ & $5.6(3.9)^{\mathrm{a}}$ & $5.2(3.8)^{a}$ & $4.9(3.9)^{\mathrm{a}}$ \\
\hline Health distress $(0-5)$ & $2.3(1.1)^{\mathrm{a}}$ & $2.1(1.1)$ & $2.0(1.0)$ & $1.7(1.2)^{\mathrm{a}}$ & $1.4(1.1)^{\mathrm{a}}$ \\
\hline Self-efficacy (0-10) & $5.5(2.1)^{a}$ & $5.9(1.9)$ & $6.1(2.1)$ & $6.1(2.3)$ & $5.9(2.8)$ \\
\hline \multicolumn{6}{|l|}{ SF-36 (0-100) } \\
\hline Mental health & $44.1(10.6)$ & $44.5(11.1)$ & $46.5(9.5)^{\mathrm{a}}$ & $47.2(10.6)^{a}$ & $47.8(10.9)^{\mathrm{a}}$ \\
\hline Role emotional & $40.5(13.4)$ & $41.5(13.2)$ & $42.6(13.4)$ & $45.3(13.1)^{\mathrm{a}}$ & $46.5(11.8)^{\mathrm{a}}$ \\
\hline Social function & $39.6(12.3)^{\mathrm{a}}$ & $41.5(12.1)$ & $42.1(11.1)$ & $42.8(13.1)$ & $43.3(11.9)$ \\
\hline Vitality & $38.8(10.3)^{\mathrm{a}}$ & $40.9(10.9)$ & $41.7(10.4)$ & $43.7(11.5)^{\mathrm{a}}$ & $43.7(12.2)^{\mathrm{a}}$ \\
\hline General health & $39.3(10.9)$ & $40.2(11.5)$ & $39.3(11.3)$ & $41.0(11.6)$ & $41.5(11.5)$ \\
\hline Bodily pain & $37.3(7.2)^{\mathrm{a}}$ & $38.9(7.6)$ & $38.8(7.5)$ & $39.8(9.0)$ & $40.6(9.4)$ \\
\hline Role physical & $32.7(10.2)^{a}$ & $35.0(11.0)$ & $36.2(12.1)$ & $39.3(13.0)^{\mathrm{a}}$ & $40.2(12.1)^{\mathrm{a}}$ \\
\hline Physical function & $34.6(11.0)$ & $34.8(11.5)$ & $35.6(11.3)$ & $37.3(11.4)^{a}$ & $38.6(10.7)^{a}$ \\
\hline
\end{tabular}

${ }^{a}$ significantly different to pre-intervention $(p<0.05)$. Repeated measures ANOVA

Decreasing scores in fatigue, pain, anxiety, depression and health distress signify improvement, while increasing scores in self-efficacy and SF-36 signify improvement

Ix Intervention

\section{Quality of life}

The SF-36 subscales of mental health, role emotional, general health and physical function remained unchanged during the pre-intervention control period, while social function, vitality, bodily pain and role physical demonstrated significant improvements. Only mental health showed a significant improvement immediately post-intervention and mental health remained significantly better than pre-intervention at both 6 and 12 months. Although there were only small non-significant improvements in role emotional and physical function at post-intervention, improvements continued over time and were statistically significant by 6 and 12 months (Table 1).

\section{Discussion}

This, one of the first studies of SM specifically for people with RA delivered by health professionals, demonstrated significant improvements in some, but not all, aspects of health and well-being measured. This results appears superior to those from non-disease specific SM programs, however, participants with RA included in generic SM arthritis studies are usually in the minority $[23,25,44,45]$, consequently presenting limitations to interpreting their results for this subset of participants. It is not clearly understood how SMIs affect change in health outcomes. Several factors that may have an affect are motivation to participate and to change health behaviour, deteriorating health, stressful life events [28], the concept of readiness to change [46, 47], and lower quality of life [40]. Our results highlight that some changes may only develop in the long term. For example improvements in anxiety, role emotional and physical function were only evident at 6 months.

Improvements in anxiety, depression and mental health are important due to their severe impact. Participants were taught how to manage the psychological impacts of RA through relaxation and distraction techniques. Improving these SM skills should enable better coping with the challenges imposed by RA [48-50]. Better knowledge and understanding of disease empowers and enhances a sense of control, and consequently people feel less anxious [7]. Armed with better behavioural and cognitive strategies to manage their disease, they are likely to be more confident, and feel less uncertain about the consequences of their disease, [48, 51], with associated improvements in mental health and depression $[49,52,53]$. Our results reflect this as participants reported experiencing fewer role limitations due to emotional problems. Barlow et al. [44] found similar improvement in anxiety and depression following participation in the Arthritis Self-Management Program (ASMP). However, no immediate post-intervention results were recorded, and results were for the combined arthritis population, therefore making it difficult to distinguish effects within the RA participants.

There was a significant improvement in health distress during the pre-intervention control period, therefore 
results need to be interpreted with caution. The improvement during the control period could be attributed to the participants' knowledge that they were joining the SMI in the near future and looking forward to a supportive environment with qualified health professionals providing reassurance. However, as for anxiety, depression and mental health, it is plausible that a greater knowledge and understanding of disease gained during the SMI, in addition to SM strategies, could reduce fear, worry and/or frustration felt in relation to RA. Other studies evaluating the effect of ASMP in an arthritis population support our findings [23, 25, 45]. Interestingly, when Lorig et al. [23] evaluated the effect on the RA population alone, no significant effect was found. Evidence from our study suggests participation in a disease-specific SMI reduces the distress associated with RA for up to 1 year.

During the program, participants were provided with relaxation and energy conservation and pacing strategies to assist with managing fatigue. It is plausible that this SMI assisted participants to establish a balance between rest and exercise resulting in overall improvements in fatigue. In addition, participants also learned to recognise fear-avoidant behaviour, which can affect physical functioning. Instead they were able to implement alternative approaches to manage activities. Fatigue is influenced by perceptions of RA and sense of control over the consequences [54]. Therefore, altering people's perceptions may subsequently mediate improvement in fatigue [49]. Other studies have reported conflicting results for improvement in fatigue. Following participation in the ASMP the reported improvement was maintained for 1 year $[23,25,46]$. However, this was not true for the RA population alone [23].

This intervention was designed to enhance patients' abilities to control their pain, and to utilise adaptive pain management strategies. However no significant reduction in pain was recorded. This finding is not unexpected. Effective pain management can be difficult to achieve in patients with RA as it relates to inflammation rather than mechanical factors. Strategies to supress inflammation, especially pharmacological, would be the most effective. Our findings are consistent with previous studies that report that many patients with RA believe their pain is uncontrollable [14], and isolated SMI may not have been sufficiently potent to change this. Moreover, pain often persisted regardless of concurrent treatment and co-management with their usual clinician based care $[7,10]$. Integration of a SM program into usual clinician based care as opposed to parallel delivery has been shown to have a positive impact on adherence to medical therapy and disease activity scores [55]. As we did not measure either of these outcomes in our study we cannot comment definitively on whether lack of improvement in disease activity may have been associated with the limited improvements in pain and fatigue observed in our study.

Changes in SE regarding pain and other symptoms relate to changes in perceived health status [56]. In people with RA there is a gradual deterioration in physical function $[6,57]$ as perpetuated by disease progression leading to disability, and impaired quality of life [58]. Patients with RA often consider their health as deteriorating, and this may be the reason for the report of no improvements in general health in the study.

Self-management interventions are often associated with improvements in SE. This is true for our study, however the improvement was not statistically significant $[23,25,46]$. Although the study used feedback questionnaires in the program to further optimise SE, there are other factors that could have affected the results [59]. Firstly the age and disease duration of the hospital cohort may have an influence as they had long disease duration and severe deformities [60]. Also the participants may have set unachievable goals and unrealistic expectations, and the subsequent failure weakened SE $[14,61]$. The small but insignificant improvement in SE is consistent with findings following an online ASMP [23]. This study's findings are also consistent with the previously reported negative association between pain ratings and perceived SE [60].

As this was a SMI study, medical interventions were not part of the assessment brief. This was managed in parallel by independent clinicians without specific knowledge of the SMI interventions and based on their independent individual patient assessments. Ongoing medical management is an important confounding variable that we were unable to control for in this study. Given the progressive destructive nature of the disease, we did not consider it ethical to withhold medical revision during the 12 month study period. Changes in medication have the potential to influence a number of the variables measured. This may in part explain the findings of significantly improved health distress, SE, bodily pain and role physical during the control period. It is plausible that better controlled pain improves SE, minimising perceived role limitations due to physical functioning, and reduced health distress. Use of DMARDS did not change overall, however bDMARDS did and may have a significant impact on outcome measures. Biologics are considered the 'ideal' and the most effective treatment in combination with a DMARD in terms of disease control and or remission. Therefore the increase in prescription rates may be considered a strength of this program as it encouraged more optimal treatment. This may be a consequence of better education and empowerment of participants. Improving education and self-efficacy in conjunction with optimisation of medical management to reduce symptoms of 
inflammation and signs of destruction would be desirable to optimise patient outcomes.

One limitation of the study is the design. A single group repeated measures design was used to evaluate short and long term benefits, so all participants could partake in the intervention. The effect of time and "any contact" with any care provider is difficult to factor in or correct for. Nevertheless, longitudinal and observational studies provide essential information about the course and outcome of rheumatic diseases that cannot be provided by randomised controlled trials [62]. However, to further develop and examine the effectiveness of the RA SMI a large randomised controlled trial would be required.

Findings from this study are important for implementing future RA SMIs. These interventions are relatively inexpensive and safe forms of treatment. They also support current health care funder priorities of shifting the management of chronic diseases into the primary care environment and the emphasis on person centred management with less reliance on specialist centres.

\section{Conclusions}

In conclusion implementing a RA SMI demonstrated significant benefits. Any contact with a supportive health care provider with a specific disease focus, appears to be beneficial for patients with RA. There appears to be additional benefit of adding health professional intervention and SMI. Improvements in depression and mental health were observed in response to the SMI. Moreover, they were maintained at 12 months. The reported increase in prescription of bDMARDS by patients' medical practitioner should be viewed as a positive and essential adjunctive strategy for disease modification and reducing impairment and disability. Empowering patients through education may allow them to be more proactive in seeking better evidence based medical treatments earlier. As this was one of the first studies on RA SMI future development and evaluation via a randomised controlled trial is necessary to continue to optimise the positive outcomes of the program. In particular refinement of the program focussing on the SE and pain outcomes needs to be planned, implemented and evaluated.

\section{Abbreviations \\ RA: Rheumatoid arthritis; SM: Self-management; SMI: Self-management intervention; EP: Education program; SE: Self-efficacy; SF-36: Short form 36; ANOVA: Analysis of variance; DMARD: Disease modifying anti rheumatic drug; bDMARD: Biologics; ASMP: Arthritis self-management program.}

\section{Competing interests}

The authors declare that they have no competing interests.

\section{Authors' contributions}

$W$ performed data analysis and interpretation, and wrote the first draft of the manuscript. KB was involved in conception and design of the study, data analysis and interpretation, writing and coordination of all suggestions and revisions to the manuscript. BL was involved in conception and design of the study, provided support during data collection and approved final version of the manuscript. Cl was involved in review of data, outcomes and interpretation and writing and revision of this paper. JMcQ was involved in conception of the study, data interpretation, in review of data, outcomes and interpretation and writing and revision of this paper. All authors participated in reviewing and editing the manuscript, and approved the final manuscript.

\section{Acknowledgements}

This program was funded by the Commonwealth Government of Australia through the Arthritis and Musculoskeletal Quality Improvement Program. The authors would like to acknowledge contributions of Christina Johnson, Jessica Rose, Jane Stott, Victoria Van, Stephanie Wilson, Arthritis and Osteoporosis Western Australia to the project and to thank all participants who made the study possible.

\section{Author details}

${ }^{1}$ School of Physiotherapy and Exercise Science, Curtin University, Perth, Western Australia, Australia. ${ }^{2}$ Department of Rheumatology, Sir Charles Gairdner Hospital, Perth, Western Australia, Australia. ${ }^{3}$ Arthritis and Osteoporosis Western Australia, Perth, Western Australia, Australia. ${ }^{4}$ School of Medicine and Pharmacology, University of Western Australia, Perth, Western Australia, Australia. ${ }^{5}$ Health, Education \& Research Program Manager, Arthritis and Osteoporosis Western Australia, PO Box 34, Wembley WA 6913, 17 Lemnos St, Shenton Park, Western Australia 6008, Australia.

Received: 4 September 2014 Accepted: 7 August 2015 Published online: 20 August 2015

\section{References}

1. Access Economics. Painful Realities: The economic impact of arthritis in Australia in 2007. Report by Access Economics for Arthritis Australia, Canberra 2007.

2. AlHW. A snapshot of arthritis in Australia 2010. In: Arthritis series no 13. Canberra: AlHW; 2010. p. 24.

3. Andersson AK, Li C, Brennan FM. Recent developments in the immunobiology of rheumatoid arthritis. Arthritis Res Ther. 2008;10(2):204.

4. Costenbader KH, Feskanich D, Mandl LA, Karlson EW. Smoking intensity, duration, and cessation, and the risk of rheumatoid arthritis in women. Am J Med. 2006:119(6):503-e501-509.

5. Arthritis and Osteoporosis Victoria. A problem worth solving: The rising costs of musculoskeletal conditions in Australia. Report by Arthritis and Osteoporosis Victoria based on analysis by Deloitte Access Economics 2013.

6. Lindqvist E, Saxne T, Geborek P, Eberhardt K. Ten year outcome in a cohort of patients with early rheumatoid arthritis: health status, disease process, and damage. Ann Rheum Dis. 2002;61(12):1055-9.

7. Scott DL, Smith C, Kingsley G. What are the consequences of early rheumatoid arthritis for the individual? Best Pract Res Clin Rheumatol. 2005;19(1):117-36

8. El-Miedany YM, El-Rasheed AH. Is anxiety a more common disorder than depression in rheumatoid arthritis? Joint Bone Spine. 2002;69(3):300-6.

9. Pollard LC, Choy EH, Gonzalez J, Khoshaba B, Scott DL. Fatigue in rheumatoid arthritis reflects pain, not disease activity. Rheumatology (Oxford). 2006:45(7):885-9.

10. Taylor P, Manger B, Alvaro-Gracia J, Johnstone R, Gomez-Reino J, Eberhardt $E$, et al. Patient perceptions concerning pain management in the treatment of rheumatoid arthritis. J Int Med Res. 2010;38(4):1213-24.

11. Ahlstrand I, Bjork M, Thyberg I, Borsbo B, Falkmer T. Pain and daily activities in rheumatoid arthritis. Disabil Rehabil. 2012;34(15):1245-53.

12. Iversen MD, Hammond A, Betteridge N. Self-management of rheumatic diseases: state of the art and future perspectives. Ann Rheum Dis. 2010;69(6):955-63.

13. Lorig KR, Holman $H$. Self-management education: history, definition, outcomes, and mechanisms. Ann Behav Med. 2003;26(1):1-7.

14. Taal E, Rasker JJ, Wiegman O. Patient education and self-management in the rheumatic diseases: a self-efficacy approach. Arthritis Care Res. 1996;9(3):229-38.

15. Mendelson AD, McCullough C, Chan A. Integrating self-management and exercise for people living with arthritis. Health Educ Res. 2011;26(1):167-77.

16. Newman S, Steed L, Mulligan K. Self-management interventions for chronic illness. Lancet. 2004:364(9444):1523-37.

17. Riemsma RP, Kirwan JR, Taal E, Rasker JJ. Patient education for adults with rheumatoid arthritis. Cochrane Database Syst Rev. 2003;2:CD003688.

18. Hammond A, Freeman K. One-year outcomes of a randomized controlled trial of an educational-behavioural joint protection programme for people with rheumatoid arthritis. Rheumatology (Oxford). 2001;40(9):1044-51. 
19. Warsi A, LaValley MP, Wang PS, Avorn J, Solomon DH. Arthritis self-management education programs: a meta-analysis of the effect on pain and disability. Arthritis Rheum. 2003;48(8):2207-13.

20. Warsi A, Wang PS, LaValley MP, Avorn J, Solomon DH. Self-management education programs in chronic disease: a systematic review and methodological critique of the literature. Arch Intern Med. 2004;164(15):1641-9.

21. Newbould J, Taylor D, Bury M. Lay-led self-management in chronic illness: a review of the evidence. Chronic IIIn. 2006;2(4):249-61.

22. Lorig KR, Ritter PL, Laurent DD, Fries JF. Long-term randomized controlled trials of tailored-print and small-group arthritis self-management interventions. Med Care. 2004;42(4):346-54.

23. Lorig KR, Ritter PL, Laurent DD, Plant K. The internet-based arthritis self-management program: a one-year randomized trial for patients with arthritis or fibromyalgia. Arthritis Rheum. 2008;59(7):1009-17.

24. Coleman S, Briffa K, Conroy H, Prince R, Carroll G, McQuade J. Short and medium-term effects of an education self-management program for individuals with osteoarthritis of the knee, designed and delivered by health professionals: a quality assurance study. BMC Musculoskelet Disord. 2008;9:117.

25. Lorig K, Ritter PL, Plant K. A disease-specific self-help program compared with a generalized chronic disease self-help program for arthritis patients. Arthritis Rheum. 2005;53(6):950-7.

26. Nunez DE, Keller $C$, Ananian CD. A review of the efficacy of the self-management model on health outcomes in community-residing older adults with arthritis. Worldviews Evid Based Nurs. 2009;6(3):130-48.

27. Solomon DH, Warsi A, Brown-Stevenson T, Farrell M, Gauthier S, Mikels D, et al. Does self-management education benefit all populations with arthritis? A randomized controlled trial in a primary care physician network. J Rheumatol. 2002;29(2):362-8

28. Bode C, Taal E, Emons PA, Galetzka M, Rasker JJ, Van de Laar MA. Limited results of group self-management education for rheumatoid arthritis patients and their partners: explanations from the patient perspective. Clin Rheumatol. 2008;27(12):1523-8.

29. Meesters JJ, Vliet Vlieland TP, Hill J, Ndosi ME. Measuring educational needs among patients with rheumatoid arthritis using the Dutch version of the Educational Needs Assessment Tool (DENAT). Clin Rheumatol. 2009;28(9):1073-7.

30. Hill J, McQuade J, Hale CA. A comparison of the educational needs of RA patients from two English speaking countries using the educational needs assessment tool (ENAT). Ann Rheum Dis. 2005;64:594-5.

31. Jensen MP, Turner JA, Romano JM. What is the maximum number of levels needed in pain intensity measurement? Pain. 1994;58(3):387-92.

32. Williamson A, Hoggart B. Pain: a review of three commonly used pain rating scales. J Clin Nurs. 2005;14(7):798-804.

33. Ferraz MB, Quaresma MR, Aquino LR, Atra E, Tugwell $P$, Goldsmith $\mathrm{CH}$. Reliability of pain scales in the assessment of literate and illiterate patients with rheumatoid arthritis. J Rheumatol. 1990;17(8):1022-4.

34. Zigmond AS, Snaith RP. The hospital anxiety and depression scale. Acta Psychiatr Scand. 1983;67(6):361-70.

35. Bjelland I, Dahl AA, Haug TT, Neckelmann D. The validity of the Hospital Anxiety and Depression Scale. An updated literature review. J Psychosom Res. 2002;52(2):69-77.

36. Lorig K, Chastain RL, Ung E, Shoor S, Holman HR. Development and evaluation of a scale to measure perceived self-efficacy in people with arthritis. Arthritis Rheum. 1989;32(1):37-44.

37. Lorig K, Stewart A, Ritter PL, Gonzalez V, Laurent DD, Lynch J. Outcome measures for health education and other health care interventions. Thousand Oakes: Sage Publications; 1996.

38. Ware JE, Sherbourne CD. The Mos 36-Item Short-Form Health Survey (Sf-36).1. Conceptual-Framework and Item Selection. Med Care. 1992;30(6):473-83.

39. Kvien TK, Kaasa S, Smedstad LM. Performance of the Norwegian SF-36 Health Survey in patients with rheumatoid arthritis. II. A comparison of the SF-36 with disease-specific measures. J Clin Epidemiol. 1998;51(11):1077-86.

40. Kingsley G, Scott IC, Scott DL. Quality of life and the outcome of established rheumatoid arthritis. Best Pract Res Clin Rheumatol. 2011;25(4):585-606.

41. McHorney CA, Ware Jr JE, Raczek AE. The MOS 36-Item Short-Form Health Survey (SF-36): II. Psychometric and clinical tests of validity in measuring physical and mental health constructs. Med Care. 1993;31(3):247-63.

42. Kosinski M, Keller SD, Hatoum HT, Kong SX, Ware Jr JE. The SF-36 Health Survey as a generic outcome measure in clinical trials of patients with osteoarthritis and rheumatoid arthritis: tests of data quality, scaling assumptions and score reliability. Med Care. 1999;37(5 Suppl):MS10-22.

43. Kosinski M, Keller SD, Ware Jr JE, Hatoum HT, Kong SX. The SF-36 Health Survey as a generic outcome measure in clinical trials of patients with osteoarthritis and rheumatoid arthritis: relative validity of scales in relation to clinical measures of arthritis severity. Med Care. 1999;37(5 Suppl):MS23-39.

44. Barlow JH, Turner AP, Wright CC. A randomized controlled study of the Arthritis Self-Management Programme in the UK. Health Educ Res. 2000;15(6):665-80

45. Goeppinger J, Lorig KR, Ritter PL, Mutatkar S, Villa F, Gizlice Z. Mail-delivered arthritis self-management tool kit: a randomized trial and longitudinal followup. Arthritis Rheum. 2009;61(7):867-75.

46. Keefe FJ, Lefebvre JC, Kerns RD, Rosenberg R, Beaupre P, Prochaska J, et al. Understanding the adoption of arthritis self-management: stages of change profiles among arthritis patients. Pain. 2000;87(3):303-13.

47. Prochaska J, DiClemente C. Towards a comprehensive, transtheoretical model of change: states of change and addictive behaviors. 2nd ed. New York: Plenum Press; 1998.

48. Sharpe L, Sensky T, Timberlake N, Ryan B, Brewin CR, Allard S. A blind, randomized, controlled trial of cognitive-behavioural intervention for patients with recent onset rheumatoid arthritis: preventing psychological and physical morbidity. Pain. 2001;89(2-3):275-83.

49. Evers AW, Kraaimaat FW, van Riel PL, de Jong AJ. Tailored cognitive-behavioral therapy in early rheumatoid arthritis for patients at risk: a randomized controlled trial. Pain. 2002;100(1-2):141-53.

50. Sharpe L, Sensky T, Timberlake N, Ryan B, Allard S. Long-term efficacy of a cognitive behavioural treatment from a randomized controlled trial for patients recently diagnosed with rheumatoid arthritis. Rheumatology (Oxford). 2003;42(3):435-41

51. Parker JC, Smarr KL, Buckelew SP, Stucky-Ropp RC, Hewett JE, Johnson JC, et al. Effects of stress management on clinical outcomes in rheumatoid arthritis. Arthritis Rheum. 1995;38(12):1807-18.

52. Astin JA, Beckner W, Soeken K, Hochberg MC, Berman B. Psychological interventions for rheumatoid arthritis: a meta-analysis of randomized controlled trials. Arthritis Rheum. 2002;47(3):291-302.

53. Sharpe L, Sensky T, Allard S. The course of depression in recent onset rheumatoid arthritis: the predictive role of disability, illness perceptions, pain and coping. J Psychosom Res. 2001;51(6):713-9.

54. Treharne GJ, Lyons AC, Hale ED, Goodchild CE, Booth DA, Kitas GD. Predictors of fatigue over 1 year among people with rheumatoid arthritis. Psychol Health Med. 2008;13(4):494-504.

55. El Miedany Y, El Gaafary M, El Arousy N, Ahmed I, Youssef S, Palmer D. Arthritis education: the integration of patient-reported outcome measures and patient self-management. Clin Exp Rheumatol. 2012;30(6):899-904.

56. Brekke M, Hjortdahl P, Kvien TK. Changes in self-efficacy and health status over 5 years: a longitudinal observational study of 306 patients with rheumatoid arthritis. Arthritis Rheum. 2003;49(3):342-8.

57. Eurenius E, Stenstrom CH. Physical activity, physical fitness, and general health perception among individuals with rheumatoid arthritis. Arthritis Rheum. 2005;53(1):48-55

58. Welsing PM, van Gestel AM, Swinkels HL, Kiemeney LA, van Riel PL. The relationship between disease activity, joint destruction, and functional capacity over the course of rheumatoid arthritis. Arthritis Rheum. 2001;44(9):2009-17.

59. Bandura A. Foundations of Thought and Action: A Social Cognitive Theory. Englewood: Prentice-Hall; 1986.

60. Lefebvre JC, Keefe FJ, Affleck G, Raezer LB, Starr K, Caldwell DS, et al. The relationship of arthritis self-efficacy to daily pain, daily mood, and daily pain coping in rheumatoid arthritis patients. Pain. 1999;80(1-2):425-35.

61. Appelbaum SH, Hare A. Self-efficacy as a mediator of goal setting and performance. J Manage Psychol. 1996;11(3):33-47.

62. Wolfe F, Lassere M, van der Heijde D, Stucki G, Suarez-Almazor M, Pincus T, et al. Preliminary core set of domains and reporting requirements for longitudinal observational studies in rheumatology. J Rheumatol. 1999;26(2):484-9. 